\title{
Caracterización de Propiedades Químicas y Fisicoquímicas de Chorizos Comercializados en la Zona Centro de México
}

\author{
Roberto González-Tenorio ${ }^{\left(1^{\star}\right)}$, Alfonso Totosaus ${ }^{(2)}$ Irma Caro $^{(3)}$ y Javier Mateo ${ }^{(3)}$ \\ (1) Universidad Autónoma del Estado de Hidalgo, Instituto de Ciencias Agropecuarias, Área Académica de \\ Ingeniería Agroindustrial, Av. Universidad s/n Km1 Rancho Universitario CP. 43600. Tulancingo, Hidalgo. \\ México (e-mail: rtenorio@uaeh.edu.mx). \\ (2) Tecnológico de Estudios Superiores de Ecatepec, Laboratorio de Alimentos, Av. Tecnológico s/n, \\ C.P. 55210 Col. Valle de Anáhuac, Ecatepec de Morelos, Estado de México-México \\ (e-mail: atotosaus@tese.edu.mx). \\ (3) Universidad de León, Facultad de Veterinaria, Dpto. de Higiene y Tecnología de los Alimentos, \\ Campus de Vegazana s/n, CP. 24071, León, España (e-mail: icarc@unileon.es; jmato@unileon.es).
}

*Autor a quien debe ser dirigida la correspondencia.

Recibido Sep. 26, 2012; Aceptado Oct. 25, 2012; Versión final recibida Dic. 14, 2012

\begin{abstract}
Resumen
Se analizaron cuarenta chorizos de cuatro tipos; dos tradicionales (de mercados rurales y carnicerías locales) y dos industriales (de centrales de abasto y supermercados) con el fin de determinar las condiciones técnicas de elaboración y comercialización de los chorizos que pueden afectar su calidad. El chorizo es un embutido crudo que se comercializa en prácticamente todo México y su elaboración se realiza tanto de modo artesanal como industrial. Se determinó el valor de $\mathrm{pH}$, la actividad de agua, el contenido de humedad, grasa, proteína, cenizas, sal y colágeno, y el perfil de ácidos grasos. Se completó la caracterización analizando la morfología de los chorizos, así como el color y textura. Se encontró variación en las características de los chorizos, dependiendo principalmente del origen de los mismos.
\end{abstract}

Palabras clave: chorizo, caracterización de alimentos, propiedades fisicoquímicas, criterios de calidad

\section{Characterization of Chemical and Physicochemical Properties of Sausages Marketed in the Central Region of Mexico}

\begin{abstract}
Forty sausages (chorizos) of four types; two traditional (rural markets and local butchers) and two industrial (wholesale large-city markets and supermarkets) were analyzed for determining the quality of the elaboration process and of the commercialization that could affect sausage quality. Chorizos are raw sausage elaborated in most places in Mexico either by small producers or by food industries. The $\mathrm{pH}$, water activity values, moisture, fat, protein, common salt and collagen contents and the fatty acid profile were determined. Additionally, morphological parameters, color and texture were assessed to complete the characterization. As expected, variations in the properties of chorizo depended on the type and origin.
\end{abstract}

Keywords: sausage, food characterization, physicochemical properties, quality criteria 


\section{INTRODUCCIÓN}

Dentro de los embutidos más populares y extendidos en toda Latinoamérica destaca el chorizo (Mateo et al., 2009). Este es un embutido típico en México, ampliamente consumido en la dieta mexicana. El chorizo mexicano es generalmente elaborado con carne y grasa de cerdo y se comercializa normalmente fresco, en cuanto a que no se madura intencionalmente (Kuri et al., 1995; Escartin et al., 1999). En el año 2010, del total del gasto familiar destinado al consumo de carne, las familias mexicanas destinaron en promedio el 83 $\%$ al consumo de carne fresca y el resto, a la compra de derivados cárnicos entre los que destacan el chorizo y longaniza (4\%), el jamón (3\%), la salchicha (2\%) y algunos otros (CMC, 2011).

De acuerdo al Consejo Mexicano de la Carne (2011), la comercialización del chorizo en México se lleva a cabo principalmente en carnicerías (41\%) y centrales de abasto mayoristas (40\%), supermercados (12\%) y mercados rurales (7\%). El lugar de comercialización se corresponde con el tipo de elaborador: grandes industrias que sustentan una marca registrada cuyos chorizos se venden en supermercados, medianas empresas de ámbito regional que elaboran y distribuyen el chorizo en centrales de abasto mayoristas, carniceros locales minoristas, y pequeños productores de ganado, en el ámbito rural y familiar. A diferencia de los dos primeros grupos, los productores de los dos últimos grupos elaboran los chorizos siguiendo formulaciones y procedimientos transmitidos de generación en generación, de forma tradicional (Guerrero et al., 2009). En su elaboración prácticamente no se usan aditivos ni ingredientes no cárnicos distintos a la sal, las especias y los condimentos, lo que está en contraste con la tendencia actual de elaborar embutidos frescos de bajo costo, con la adición de proteínas no cárnicas y altos niveles de grasa, así como diversos aditivos (fosfatos, conservantes, saborizantes, colorantes, etc.), sustancias de relleno (almidones y gomas) y/o azúcares (Feiner, 2006).

Aunque los embutidos frescos (como el chorizo) se deben almacenar y comercializar en condiciones de refrigeración (Feiner, 2006), la capacidad de muchos productores y vendedores locales de mantener la cadena de frío es limitada. De esta forma, durante el oreo y la comercialización, los chorizos se mantienen a un rango diverso de temperaturas (no controladas) durante un periodo de tiempo que puede variar desde un día hasta unas pocas semanas. Durante este periodo de tiempo, puede tener lugar una fermentación y un secado espontáneos (Kuri et al., 1995), que modifican las propiedades iniciales de los chorizos.

A pesar de la popularidad del chorizo en México, en general son pocos los estudios encontrados sobre su composición química (Pérez et al., 1999 y Austria et al., 2006). De acuerdo a estos autores, los chorizos analizados se caracterizaron por un valor de $\mathrm{pH}$ comprendido entre 4,2 y 5,1 , de $\mathrm{a}_{\mathrm{w}}$ entre 0,94 y 0,98 , de humedad $45,1-56,4 \%$, y proteína, grasa y cenizas sobre extracto seco de $31,6-40,3 \%, 48,2-56,9 \%$ y 3,8$5,1 \%$, respectivamente.

Debido a que la información científica sobre el chorizo mexicano es escasa y considerando el interés social y comercial de la caracterización de productos típicos y tradicionales en Latinoamérica, el objetivo de este estudio fue determinar diversas propiedades químicas y fisicoquímicas en el chorizo de interés sobre la definición del producto y su calidad. Se ha contrastado los valores de esas propiedades entre los diferentes tipos de chorizos que se consumen en el centro de México (agrupados en función del tipo de elaborador y modo de comercialización) con el fin de establecer diferencias y contribuir así a estandarizar su calidad. Este estudio se complementa con otros dos donde se determina la composición mineral y microbiológica de esos tipos de chorizos (González-Tenorio et al., 2012a y b).

\section{MATERIALES Y METODOS}

\section{Recolección de muestras}

En el estudio se llevó a cabo un muestreo aleatorio dirigido. Se recolectaron 40 muestras de 1 a $2 \mathrm{~kg}$ de cada una, 10 muestras de cada uno de los siguientes cuatro tipos de productores y en los siguientes puntos de venta: i) productores rurales en mercados locales rurales, ii) carniceros minoristas en carnicerías locales, iii) productores mayoristas regionales en centrales de abasto y iv) industrias de ámbito nacional con marca registrada en supermercados. Todas las muestras se encontraban expuestas al público, colgadas, sin envasado y a temperatura ambiente. Una vez adquiridas, las muestras fueron empacadas en bolsas de polietileno y transportadas en contenedores aislantes y almacenadas en refrigeración para su posterior análisis. Para los chorizos de mercados rurales fueron elegidos al azar 10 municipios del Estado de Hidalgo de menos de 10,000 habitantes y se muestreó en un puesto del mercado rural de cada municipio; para tomar la muestra como válida el chorizo tuvo que ser elaborado por un productor de cerdos en el ámbito local que no tuviese carnicería. Los chorizos de carnicerías, se adquirieron en 10 colonias (barrios) de la ciudad de Tulancingo, Hidalgo elegidas al azar; en cada colonia fue elegida una carnicería de las más conocidas y con elevado volumen de ventas, cuya selección se hizo a través de preguntas a vecinos y 
conocidos implicados en el sector; todos los chorizos fueron elaborados en las propias carnicerías. Los chorizos comercializados en centrales de abasto mayoristas se tomaron en 10 puestos de las centrales mayoristas de las dos grandes ciudades del Estado de Hidalgo, Pachuca y Tulancingo; de cada ciudad se eligieron los negocios con mayor volumen de ventas de chorizos (particularmente los que venden chorizos más económicos debido a la gran competencia por precio en este tipo de mercados). Finalmente, en el caso de los chorizos de centro comercial, se muestrearon prácticamente todas las marcas de chorizo con presencia en el ámbito nacional y con registro sanitario, encontradas en los distintos centros comerciales de Tulancingo y Pachuca.

$\mathrm{pH}$, humedad, actividad de agua, sal y cenizas

La medida del pH se llevó a cabo según el método descrito por Guerrero y Arteaga (1990) utilizando un pHmetro (Termo Orión; 420A, Beverly, MA., EEUU). El contenido de humedad de acuerdo al método 950.46 de la AOAC, mediante secado en horno de convección de aire forzado. La actividad de agua se determinó utilizando un equipo Aqua Lab; 3TE (Decagon Devices, Inc., Pullman, WA., EEUU), a temperatura ambiente $\left(25 \pm 2{ }^{\circ} \mathrm{C}\right)$. El contenido de $\mathrm{Na}$ de acuerdo al método 986.09 mediante espectroscopia de emisión atómica acoplada inductivamente (ICP-AES) utilizando un espectrofotómetro Perkin Elmer (Optima 2000 DV) Emission Spectrometry. Por último la determinación de cenizas según el método 920.153. A partir del contenido en $\mathrm{Na}$ se estimó la cantidad de sal considerando la totalidad del $\mathrm{Na}$ como $\mathrm{NaCl}$.

Proteína total, colágeno, grasa, ácidos grasos totales y ácidos orgánicos

La determinación de proteína se realizó cuantificando el nitrógeno total según el método Kjeldahl siguiendo el procedimiento 992.15. La determinación del contenido de colágeno se realizó de acuerdo al protocolo de Hill (1976). En la determinación del contenido de grasa se utilizó el sistema de extracción de grasa Soxhlet automatizado (Büchi, B-811, Flawil, Suiza) siguiendo la metodología descrita en 991.36 (AOAC,1999). La determinación de los ácidos grasos totales se realizó mediante el método de trans-esterificación in situ y análisis de los metil esteres de los ácidos grasos por cromatografía gaseosa acoplada a espectrometría de masas. Para la metilación de los ácidos grasos se utilizó el protocolo de Carrapiso et al. (2000). En la cuantificación de los ácidos grasos se utilizó un cromatógrafo de gases (HP 6890 Series GC system, Ramsey, MN., EEUU), acoplado a un detector selectivo de masas (HP 5973 Inert MSD Mass Selective Detector) equipado con un inyector automático (HP 7683 series inyector). Los metil ésteres de los ácidos grasos fueron separados con una columna capilar (Omegawax 250 Fused Silica; Supelco, Madrid, España), de $30 \mathrm{~m}$ de longitud $\times 0.250 \mathrm{~mm}$ de diámetro interno y grosor de la película de $0.25 \mu \mathrm{m}$. La identificación se llevó a cabo por comparación de los tiempos de retención con los patrones externos (Sigma-Aldrich) y posterior comprobación con los espectros de masas de los picos con los ácidos grasos de la base de datos HP Willey 275. L Mass Spectral Library (HP, revisión D 01.00, 1998) proporcionada por el fabricante.

\section{Ácidos orgánicos de cadena corta}

El contenido en ácidos orgánicos se determinó de acuerdo al método descrito por Bruna et al. (2003), utilizando un cromatógrafo (Alliance-Waters 2690; Waters Corporation, Milford, MA, EEUU), equipado con un detector de refractometría (Waters 410), una columna de separación de intercambio iónico Bio RadAminex (HPX-87H) de longitud $300 \mathrm{~mm} \times 7.8 \mathrm{~mm}$, protegida con una columna Micro-Guard H+ (Bio-Rad Laboratorios, Hércules, CA., EEUU) de $3 \mathrm{~cm}$ x $4.6 \mathrm{~mm}$. Se empleó un detector "Diode Array" (Waters 996).

\section{Características morfológicas, de color y textura}

Las determinaciones de las dimensiones y tamaño de grano de los chorizos se realizaron mediante la medición del diámetro del embutido y el tamaño de la partícula de carne y grasa, realizando 10 mediciones de partícula por cada tipo de chorizo. La determinación del color de los chorizos en coordenadas CIE-Lab, se realizó utilizando un espectro colorímetro de reflectancia CM-508d marca Minolta (Osaka, Japón), con un ángulo del observador de $2^{\circ}$, un iluminante $\mathrm{D} 65$ y en modo SCl. La textura se determinó mediante un análisis del perfil de textura sobre cubos de chorizo de $1,5 \mathrm{~cm}$ de lado obtenidos de la parte más central de los mismos. Se utilizó un equipo Texture Analyser modelo TA-HDi (Texture Technologies, Scarsdale/ NY Stable Micro System, Surrey, England). Las condiciones de medida fueron $50 \%$ de compresión, velocidad de la sonda (25 mm de diámetro) de $1 \mathrm{~mm} / \mathrm{s}$, fuerza de carga de $5 \mathrm{~kg}$. De las curvas fuerza-tiempo se determinó: dureza, adhesividad, cohesividad, elasticidad y masticabilidad (Szcesniak, 1963; Bourne, 1978).

\section{Análisis estadístico}

Los análisis estadísticos se llevaron a cabo con el software Statistica for Windows (versión 6.0), Statsoft Inc. (Tulsa, OK., EEUU). Se empleó una estadística descriptiva básica, determinando el valor medio y la 
desviación estándar para cada parámetro evaluado, así como un análisis de varianza (ANOVA) de una vía para identificar diferencias entre los distintos tipos de chorizos $(P<0.05)$.

\section{RESULTADOS Y DISCUSIÓN}

\section{Composición proximal}

La Tabla 1 muestra los resultados de los componentes mayoritarios de los chorizos analizados. Se observó una alta variabilidad en los distintos componentes con coeficientes de variación entre 10 y $40 \%$. La mayor variación se encontró en chorizos de mercados rurales, el componente de mayor variabilidad fue el de proteína sobre materia seca. La menor variabilidad se presentó en los chorizos de centrales de abasto y centros comerciales. La variabilidad observada puede atribuirse a diferencias en el grado de secado o al tipo y las cantidades de carne y grasa utilizados en la formulación.

Tabla 1. Composición proximal (\%) de los chorizos analizados. Medias con el mismo superíndice en el mismo renglón no son significativamente diferentes $(p<0.05)$

\begin{tabular}{|l|c|c|c|c|}
\hline \multicolumn{1}{|c|}{ Composición Proximal } & $\begin{array}{c}\text { Carnicerías } \\
(\mathrm{n}=10)\end{array}$ & $\begin{array}{c}\text { Mercados } \\
\text { rurales }(\mathrm{n}=10)\end{array}$ & $\begin{array}{c}\text { Centros comerciales } \\
(\mathrm{n}=10)\end{array}$ & $\begin{array}{c}\text { Centrales de } \\
\text { abasto }(\mathrm{n}=10)\end{array}$ \\
\hline Humedad & $42.89 \pm 9.49$ & $42.68 \pm 13.57$ & $36.47 \pm 8.75$ & $38.72 \pm 6.54$ \\
\hline Proteína (materia seca) & $29.63 \pm 9.44^{\mathrm{ab}}$ & $37.79 \pm 14.05^{\mathrm{a}}$ & $30.87 \pm 3.81^{\mathrm{a}}$ & $21.95 \pm 5.69^{\mathrm{b}}$ \\
\hline Grasa (materia seca) & $60.07 \pm 8.55^{\mathrm{ab}}$ & $53.25 \pm 13.46^{\mathrm{b}}$ & $52.18 \pm 8.38^{\mathrm{b}}$ & $64.85 \pm 8.37^{\mathrm{a}}$ \\
\hline Cenizas (materia seca) & $5.02 \pm 1.27^{\mathrm{b}}$ & $5.22 \pm 1.53^{\mathrm{b}}$ & $7.33 \pm 1.49^{\mathrm{a}}$ & $5.32 \pm 1.30^{\mathrm{b}}$ \\
\hline Cocientes & & & & \\
\hline Sal/Humedad & $0.054 \pm 0.022^{\mathrm{b}}$ & $0.068 \pm 0.065^{\mathrm{b}}$ & $0.119 \pm 0.068^{\mathrm{a}}$ & $0.070 \pm 0.020^{\mathrm{b}}$ \\
\hline Humedad/Proteína & $2.68 \pm 0.59^{\mathrm{ab}}$ & $2.15 \pm 0.70^{\mathrm{bc}}$ & $1.95 \pm 0.69^{\mathrm{c}}$ & $2.96 \pm 0.33^{\mathrm{a}}$ \\
\hline
\end{tabular}

Respecto a la humedad y el grado de secado, la mayoría de los chorizos de carnicerías y mercados rurales se pueden incluir en la categoría de embutidos semisecos por tener un porcentaje de humedad entre el 40 y $50 \%$ y una relación humedad proteína entre 2.3 y 3.7 (Adams, 1986). Los chorizos de centrales de abasto también se pueden considerar semisecos ya que, a pesar de tener una humedad ligeramente inferior al $40 \%$, presentaron una relación humedad/proteína elevada (similar a 3), lo que se justifica por su alto contenido en grasa. De acuerdo a Adams (1986), las pérdidas de peso para este tipo de embutidos se estiman entre el 10 y $20 \%$. Sin embargo, la mayor parte de los chorizos de centros comerciales presentaron una humedad inferior al $40 \%$ y una relación humedad proteína menor a 2.3 por lo que se podrían considerar como embutidos secos. Se estima que en estos productos las pérdidas de humedad durante el secado son superiores al 20\%. Las diferencias en el grado de secado entre tipos de chorizo se reflejan en la diferencias significativas encontradas para la relación humedad/proteína $(P<0.01)$, valores inferiores en chorizos de centros comerciales y superiores en los de centrales de abasto. Según Prieto y Carballo (1997), otro parámetro utilizado para evaluar el grado de secado de un embutido es el cociente entre peso de la humedad sobre el peso de embutido desengrasado (H/ED). El H/ED de la carne fresca se estima en 0.77 y el encontrado en los chorizos fue de 0.66 (carnicerías), 0.63 (mercados rurales), 0.55 (centros comerciales), 0.65 (centrales de abasto). Según estos datos, el chorizo de centros comerciales es el que mayor grado de secado experimenta. Los resultados obtenidos en los chorizos mexicanos considerados como semisecos están en general dentro de los rangos encontrados en otros estudios o normativas de chorizos, salchichas o longanizas latinoamericanos, como es el caso de algunos chorizos y salchichas peruanos (Reyes-García et al., 2009; Ramos, et al., 2011) o mexicanos (Austria et al., 2006).

Los chorizos con mayor valor de proteína sobre materia seca fueron los de mercados rurales y los de menor valor los de centrales de abasto. Tanto los porcentajes de proteína como los de grasa dan una idea clara de que los productores rurales elaboran el chorizo con mayor relación carne/grasa en contraste con los elaboradores de centrales de abasto. La cantidad de proteína influye en la calidad de los chorizos, debido a que ésta permite mejores propiedades funcionales de ligado de la grasa, sin dejar de mencionar la importancia que tiene sobre el aspecto nutritivo.

El porcentaje de grasa sobre materia fresca no presentó diferencias significativas entre tipos de chorizos aunque los chorizos de centrales de abasto fueron los de mayor contenido graso. Este tipo de chorizos presentó el mayor porcentaje en grasa sobre materia seca, diferenciándose significativamente (Tabla 1; $P<0.05)$ de los chorizos de mercados rurales y centros comerciales, con el menor contenido en grasa. En la calidad de los chorizos, la grasa es una propiedad con repercusiones importantes debido a que está involucrada directamente el sabor, textura, valor nutritivo y estabilidad oxidativa. 
El contenido en cenizas en los chorizos analizados (Tabla 1) varió entre el $5.0 \%$ y el 7.3 \%, observándose diferencias significativas. Estas diferencias se pueden atribuir a la mayor adición de sales (principalmente $\mathrm{NaCl}$ ) en los chorizos de centros comerciales.

$\mathrm{pH}$, actividad de agua, sal, colágeno y ácidos orgánicos

La masa cárnica recién embutida siempre que no se usen acidulantes, tiene un pH alrededor de 5.8 y una $\mathrm{a}_{\mathrm{w}}$ elevada en torno a 0.96 , lo que se traduce en condiciones bastante idóneas para el crecimiento microbiano (Reuter, 1981; Frey, 1995). La reducción de la $\mathrm{a}_{\mathrm{w}}$ por desecación y el descenso del pH por fermentación son responsables de que los chorizos adquieran su capacidad de conservación a temperatura ambiente, toda vez que las bacterias responsables de la descomposición son incapaces de multiplicarse con bajos valores de pH y $\mathrm{a}_{\mathrm{w}}$ (Frey, 1995; CFIA, 2007). El pH en los embutidos es importante debido a que valores superiores a 6.0 pueden ocasionar el desarrollo de bacterias alterantes del producto durante su secado o conservación y pH bajos (< a 4.5) pueden ser responsables de sabores ácidos y desagradables al consumidor (Reuter, 1981; Frey, 1995).

Tabla 2. Valores de $\mathrm{pH}, \mathrm{a}_{\mathrm{w}}$, contenidos de sal, colágeno y ácidos orgánicos de los chorizos analizados. Medias con el mismo superíndice en el mismo renglón no son significativamente diferentes $(p<0.05)$

\begin{tabular}{|l|c|c|c|c|}
\hline & Carnicerías $(\mathrm{n}=10)$ & $\begin{array}{c}\text { Mercados } \\
\text { rurales }(\mathrm{n}=10)\end{array}$ & $\begin{array}{c}\text { Centros comerciales } \\
(\mathrm{n}=10)\end{array}$ & $\begin{array}{c}\text { Centrales de } \\
\text { abasto }(\mathrm{n}=10)\end{array}$ \\
\hline $\mathrm{pH}$ & $5.06 \pm 0.27$ & $5.01 \pm 0.25$ & $5.36 \pm 0.51$ & $5.10 \pm 0.47$ \\
\hline $\mathrm{a}_{\mathrm{w}}$ & $0.957 \pm 0.02^{\mathrm{a}}$ & $0.938 \pm 0.08^{\mathrm{ab}}$ & $0.899 \pm 0.08^{\mathrm{b}}$ & $0.948 \pm 0.02^{\mathrm{ab}}$ \\
\hline Sal (g/100g materia seca) & $3.78 \pm 0.89^{\mathrm{b}}$ & $3.75 \pm 1.16^{\mathrm{b}}$ & $6.10 \pm 1.67^{\mathrm{a}}$ & $4.39 \pm 1.30^{\mathrm{b}}$ \\
\hline Colágeno (g/100 g proteína) & $14.01 \pm 6.41$ & $11.46 \pm 4.87$ & $13.92 \pm 9.07$ & $19.80 \pm 14.28$ \\
\hline Ácido láctico (mg/100g) & $1383 \pm 347.2$ & $1644.9 \pm 222.7$ & $1270.0 \pm 740.5$ & $1295.7 \pm 608.8$ \\
\hline Ácido acético $(\mathrm{mg} / 100 \mathrm{~g})$ & $60.8 \pm 46.8^{\mathrm{b}}$ & $450.3 \pm 456.4^{\mathrm{a}}$ & $227.9 \pm 348.8^{\mathrm{ab}}$ & $57.8 \pm 67.7^{\mathrm{b}}$ \\
\hline
\end{tabular}

En la Tabla 2 se muestran los valores de $\mathrm{pH}$ de los distintos tipos de chorizos, los cuales estuvieron comprendidos entre 5.0 y 5.4 sin detectarse diferencias significativas entre tipos. Estos valores permiten clasificar a la mayoría de los chorizos analizados como embutidos de alta acidificación $(\mathrm{pH}<5.3)$, (Incze, 1998). Los bajos valores de $\mathrm{pH}$ se pueden atribuir al menos parcialmente al aporte de azúcares fermentables por parte del chile utilizado en la formulación y a su fermentación. En chorizos españoles el pimentón utilizado aporta cantidades de azúcares, entre 2 y 3 g por kilo (Aguirrezabal, et al., 1998), cantidades que de acuerdo a Vösgen (1995), podrían producir descensos en el pH de 0.2 a 0.3 . No obstante, con los valores de pH de los chorizos muestreados cabría esperar una mayor producción de ácido láctico que la derivada de los azúcares del pimentón o chile. El efecto sobre el pH de la eventual adición de vinagre y el alto contenido en grasa (menor capacidad tampón de la masa cárnica) del chorizo podría contribuir a explicar esta aparente contradicción.

En relación a la $a_{w}$, se observaron diferencias significativas entre los tipos de chorizo analizados (Tabla 2; $P<0.05)$. Los chorizos que mayor $a_{w}$ tuvieron, fueron los de carnicerías (0.957) mercados rurales (0.938) y centrales de abasto (0.948) y los de $a_{w}$ menor los de centros comerciales (0.899). El bajo valor de $a_{w}$ de estos últimos se puede atribuir al relativamente alto contenido en sal y bajo en humedad. En cuanto al contenido de sal se observaron diferencias significativas. El mayor contenido (Tabla $2 ; P<0.001$ ) lo tuvieron los chorizos de centros comerciales con un 6.10\% sobre MS. No existieron diferencias entre los demás tipos de chorizos, los cuales tuvieron alrededor de un 3.7 y $4.4 \%$ (MS). Este comportamiento concuerda con el encontrado para el contenido en cenizas. Es por tanto un hecho que los chorizos catalogados como "de centros comerciales" Ilevan más sal en la formulación que el resto. La sal, es un ingrediente que favorece la solubilización de las proteínas miofibrilares y mejora la estabilidad microbiológica (Feiner, 2006) pero su consumo en exceso puede ser perjudicial.

El contenido en colágeno sobre proteína total es un indicador de la cantidad de tendones o incluso cortezas que hayan sido incorporadas a la masa cárnica del chorizo. Los chorizos de centrales de abasto (Tabla 2) presentaron el mayor contenido en colágeno sobre proteína total, aunque las diferencias con los otros tipos de chorizo no fueron significativas (gran dispersión en los valores obtenidos en los chorizos de este tipo). En chorizos fermentados europeos se han descrito valores entre 1600-2500 mg de lactato por $100 \mathrm{~g}$ de materia seca (Mateo, et al., 1996; Demeyer, et al., 2000). Los chorizos de mercados rurales se sitúan dentro del rango por lo que cabe pensar que en la mayor parte de estos hubo una fermentación considerable, mientras que en los otros tipos la fermentación fue en general de menor intensidad ya que el contenido en lactato fue menor. La cantidad de ácido acético producido como metabolito secundario de la fermentación de tipo heteroláctica de los azúcares en embutidos fermentados, suele estar comprendida entre el 5 y el $10 \%$ de la cantidad de ácido láctico (Mateo, et al., 1996; Demeyer, et al., 2000). Los chorizos de carnicerías y los de centrales de abasto cumplen con esta premisa y son los de menor contenido en ácido acético. Por el 
contrario, en los chorizos de mercados rurales y centros comerciales, el contenido de ácido acético fue del 20-30\% respecto al contenido de ácido láctico. Esta cifra induce a decir que en estos tipos de chorizo es frecuente la adición de vinagre como fuente de ácido acético en la formulación.

\section{Ácidos grasos totales}

En la Tabla 3 se muestran los resultados de los porcentajes de ácidos grasos detectados para cada tipo de chorizo y en la Tabla 4 los ácidos grasos según el grado de saturación, de ácidos grasos de cadena impar y ramificada según el tipo de chorizo. Independientemente del tipo de chorizo, los ácidos grasos mayoritarios en orden de abundancia fueron C18:1, C16:0, C18:2, C18:0, C16:1, C14:0 y C18:3, siendo los niveles de C18:0 y C18:2 muy similares. Este orden de abundancia y los porcentajes obtenidos en estos ácidos grasos concuerdan en gran medida con los valores de la carne de cerdo blanco recogidos en la base de datos USDA (2010) o en los trabajos de Wood et al. (2004 y 2008).

Las diferencias significativas más reseñables entre tipos de chorizo (Tabla 4) se establecen entre chorizos de carnicerías y el resto. Los chorizos de carnicerías tuvieron más porcentaje de C14:0, C18:0 y C16:1 que el resto, así como un menor porcentaje de C18:2. Las cantidades de ácidos grasos ramificados y de cadena impar encontradas en grasa de vacuno están entre 1-3\% y 2-6\%, respectivamente (Raes, et al., 2004; Aldai, et al., 2007). Teniendo en cuenta estas cantidades y las diferencias entre los valores encontrados en los chorizos de carnicerías y los otros chorizos, es posible que la grasa de vacuno esté presente en el chorizo de carnicerías y se sitúe dentro del rango del $20-50 \%$ de la grasa total, grasa que puede provenir a partir de los recortes obtenidos durante el acondicionamiento de las piezas cárnicas. No obstante, las diferencias encontradas entre chorizos de carnicerías y el resto de tipos son moderadas y no parecen ser suficientes como para causar una clara variación en el aroma de los chorizos o tengan una repercusión importante en la calidad nutritiva.

A este respecto, los productos de origen animal como el chorizo son una fuente importante de energía aportando grasa y proteína a la dieta, aunque las grasas saturadas están asociadas como un factor de alto riesgo de enfermedades cardiovasculares, diabetes, algunos tipos de cáncer y otras enfermedades degenerativas, por lo que se recomienda moderar su ingesta ya que la grasa es a menudo señalada como la mayor fuente de exceso de energía ligada al incremento epidémico de sobrepeso y obesidad (Walker et al., 2005; Chang y Chow, 2008). Desde un punto de vista nutritivo no hay grandes diferencias entre tipos de chorizo en el perfil lipídico de su grasa, salvo tal vez la desventaja del menor contenido en ácidos grasos poliinsaturados en los chorizos de carnicerías. También hay que tener en cuenta que los de centrales de abasto presentaron mayor cantidad de grasa.

Tabla 3. Contenido (\%) en ácidos grasos de los cuatro tipos de chorizos evaluados. Medias con el mismo superíndice en el mismo renglón no son significativamente diferentes $(p<0.05)$

\begin{tabular}{|c|c|c|c|c|}
\hline Ácidos grasos & $\begin{array}{c}\text { Carnicerías } \\
(\mathrm{n}=10)\end{array}$ & $\begin{array}{c}\text { Mercados rurales } \\
(\mathrm{n}=10)\end{array}$ & $\begin{array}{c}\text { Centros comerciales } \\
(\mathrm{n}=10)\end{array}$ & $\begin{array}{c}\text { Centrales de } \\
\text { abasto }(\mathrm{n}=10)\end{array}$ \\
\hline $\mathrm{C} 10: 0$ & $0.08 \pm 0.02^{\mathrm{b}}$ & $0.09 \pm 0.02^{\mathrm{ab}}$ & $0.11 \pm 0.01^{\mathrm{a}}$ & $0.11 \pm 0.04^{\mathrm{a}}$ \\
\hline $\mathrm{C} 12: 0$ & $0.12 \pm 0.03$ & $0.12 \pm 0.06$ & $0.14 \pm 0.09$ & $0.11 \pm 0.03$ \\
\hline $\mathrm{C} 14: \mathrm{r}$ & $0.03 \pm 0.04^{\mathrm{a}}$ & $0.01 \pm 0.01^{\mathrm{b}}$ & $0.00 \pm 0.00^{\mathrm{b}}$ & $0.01 \pm 0.02^{\mathrm{b}}$ \\
\hline $\mathrm{C} 14: 0$ & $1.90 \pm 0.52^{\mathrm{a}}$ & $1.52 \pm 0.14^{\mathrm{b}}$ & $1.53 \pm 0.12^{\mathrm{b}}$ & $1.46 \pm 0.10^{\mathrm{b}}$ \\
\hline $\mathrm{C} 15: \mathrm{r}$ & $0.19 \pm 0.21^{\mathrm{a}}$ & $0.04 \pm 0.07^{\mathrm{b}}$ & $0.00 \pm 0.00^{\mathrm{b}}$ & $0.00 \pm 0.00^{\mathrm{b}}$ \\
\hline $\mathrm{C} 15: 1$ & $0.25 \pm 0.31^{\mathrm{a}}$ & $0.05 \pm 0.05^{\mathrm{b}}$ & $0.01 \pm 0.02^{\mathrm{b}}$ & $0.01 \pm 0.01^{\mathrm{b}}$ \\
\hline $\mathrm{C} 15: 0$ & $0.25 \pm 0.24^{\mathrm{a}}$ & $0.09 \pm 0.07^{\mathrm{b}}$ & $0.05 \pm 0.01^{\mathrm{b}}$ & $0.05 \pm 0.01^{\mathrm{b}}$ \\
\hline $\mathrm{C} 16: \mathrm{r}$ & $0.12 \pm 0.13^{\mathrm{a}}$ & $0.02 \pm 0.04^{\mathrm{b}}$ & $0.00 \pm 0.00^{\mathrm{b}}$ & $0.00 \pm 0.00^{\mathrm{b}}$ \\
\hline $\mathrm{C} 16: 0$ & $20.53 \pm 1.28$ & $20.79 \pm 1.20$ & $20.51 \pm 0.71$ & $20.28 \pm 0.61$ \\
\hline $\mathrm{C} 17: \mathrm{r}$ & $0.20 \pm 0.21^{\mathrm{a}}$ & $0.07 \pm 0.05^{\mathrm{b}}$ & $0.02 \pm 0.03^{\mathrm{b}}$ & $0.04 \pm 0.02^{\mathrm{b}}$ \\
\hline $\mathrm{C} 16: 1$ & $3.48 \pm 0.76^{\mathrm{a}}$ & $3.05 \pm 0.47^{\mathrm{b}}$ & $2.98 \pm 0.22^{\mathrm{b}}$ & $2.67 \pm 0.37^{\mathrm{b}}$ \\
\hline $\mathrm{C} 17: 0$ & $0.82 \pm 0.40^{\mathrm{a}}$ & $0.56 \pm 0.20^{\mathrm{b}}$ & $0.43 \pm 0.09^{\mathrm{b}}$ & $0.48 \pm 0.10^{\mathrm{b}}$ \\
\hline $\mathrm{C} 18: \mathrm{r}$ & $0.07 \pm 0.08^{\mathrm{a}}$ & $0.01 \pm 0.02^{\mathrm{b}}$ & $0.00 \pm 0.00^{\mathrm{b}}$ & $0.00 \pm 0.00^{\mathrm{b}}$ \\
\hline $\mathrm{C} 17: 1$ & $0.56 \pm 0.18^{\mathrm{a}}$ & $0.44 \pm 0.13^{\mathrm{b}}$ & $0.36 \pm 0.06^{\mathrm{b}}$ & $0.37 \pm 0.04^{\mathrm{b}}$ \\
\hline $\mathrm{C} 18: 0$ & $13.81 \pm 1.96^{\mathrm{a}}$ & $12.67 \pm 1.34^{\mathrm{ab}}$ & $11.72 \pm 0.85^{\mathrm{b}}$ & $12.96 \pm 1.94^{\mathrm{ab}}$ \\
\hline $\mathrm{C} 18: 1$ & $42.49 \pm 1.73$ & $42.56 \pm 2.75$ & $42.18 \pm 1.21$ & $42.23 \pm 1.54$ \\
\hline $\mathrm{C} 18: 2$ & $11.26 \pm 3.46^{\mathrm{b}}$ & $13.60 \pm 2.20^{\mathrm{a}}$ & $15.58 \pm 1.26^{\mathrm{a}}$ & $14.87 \pm 1.26^{\mathrm{a}}$ \\
\hline $\mathrm{C} 19: 1$ & $0.03 \pm 0.06$ & $0.04 \pm 0.08$ & $0.04 \pm 0.06$ & $0.07 \pm 0.06$ \\
\hline $\mathrm{C} 20: 0$ & $0.37 \pm 0.06$ & $0.36 \pm 0.16$ & $0.44 \pm 0.09$ & $0.44 \pm 0.10$ \\
\hline $\mathrm{C} 18: 3$ & $0.80 \pm 0.13$ & $0.83 \pm 0.11$ & $0.85 \pm 0.08$ & $0.85 \pm 0.10$ \\
\hline
\end{tabular}


Tabla 3: Continuación

\begin{tabular}{|c|c|c|c|c|}
\hline $\mathrm{C} 20: 1$ & $1.01 \pm 0.11^{\mathrm{b}}$ & $1.07 \pm 0.16^{\mathrm{ab}}$ & $1.04 \pm 0.07^{\mathrm{ab}}$ & $1.15 \pm 0.11^{\mathrm{a}}$ \\
\hline CLA C18:2 & $0.11 \pm 0.07^{\mathrm{a}}$ & $0.05 \pm 0.04^{\mathrm{b}}$ & $0.05 \pm 0.04^{\mathrm{b}}$ & $0.05 \pm 0.04^{\mathrm{b}}$ \\
\hline $\mathrm{C} 20: 2$ & $0.56 \pm 0.17^{\mathrm{b}}$ & $0.66 \pm 0.10^{\mathrm{a}}$ & $0.72 \pm 0.06^{\mathrm{a}}$ & $0.74 \pm 0.04^{\mathrm{a}}$ \\
\hline $\mathrm{C} 20: 3$ & $0.24 \pm 0.07$ & $0.28 \pm 0.15$ & $0.25 \pm 0.01$ & $0.24 \pm 0.02$ \\
\hline $\mathrm{C} 20: 4$ & $0.49 \pm 0.24^{\mathrm{b}}$ & $0.78 \pm 0.41^{\mathrm{a}}$ & $0.74 \pm 0.08^{\mathrm{ab}}$ & $0.58 \pm 0.25^{\mathrm{ab}}$ \\
\hline $\mathrm{C} 24: 0$ & $0.05 \pm 0.02$ & $0.05 \pm 0.03$ & $0.06 \pm 0.01$ & $0.05 \pm 0.01$ \\
\hline $\mathrm{C} 22: 4$ & $0.14 \pm 0.04^{\mathrm{b}}$ & $0.17 \pm 0.05^{\mathrm{ab}}$ & $0.19 \pm 0.01^{\mathrm{a}}$ & $0.18 \pm 0.04^{\mathrm{ab}}$ \\
\hline
\end{tabular}

Tabla 4. Sumatorias de las distintas clases de ácidos grasos (\%) en cada tipo de chorizo. Medias con el mismo superíndice en el mismo renglón no son significativamente diferentes $(p<0.05)$

\begin{tabular}{|l|c|c|c|c|}
\hline & $\begin{array}{c}\text { Carnicerías } \\
(n=10)\end{array}$ & $\begin{array}{c}\text { Mercados } \\
\text { rurales }(n=10)\end{array}$ & $\begin{array}{c}\text { Centros comerciales } \\
(n=10)\end{array}$ & $\begin{array}{c}\text { Centrales de } \\
\text { abasto }(n=10)\end{array}$ \\
\hline Saturados & $37.95 \pm 3.34^{\mathrm{a}}$ & $36.25 \pm 2.03^{\mathrm{ab}}$ & $35.00 \pm 1.45^{\mathrm{b}}$ & $35.94 \pm 2.50^{\mathrm{ab}}$ \\
\hline Monoinsaturados & $48.45 \pm 2.02$ & $47.37 \pm 3.13$ & $46.63 \pm 1.45$ & $46.55 \pm 1.86$ \\
\hline Poliinsaturados & $13.61 \pm 3.74^{\mathrm{b}}$ & $16.37 \pm 2.69^{\mathrm{a}}$ & $18.37 \pm 1.37^{\mathrm{a}}$ & $17.50 \pm 1.59^{\mathrm{a}}$ \\
\hline Ramificados & $0.61 \pm 0.65^{\mathrm{a}}$ & $0.15 \pm 0.19^{\mathrm{b}}$ & $0.02 \pm 0.03^{\mathrm{b}}$ & $0.04 \pm 0.03^{\mathrm{b}}$ \\
\hline Impares & $2.31 \pm 1.46^{\mathrm{a}}$ & $1.29 \pm 0.51^{\mathrm{b}}$ & $0.92 \pm 0.21^{\mathrm{b}}$ & $1.04 \pm 0.15^{\mathrm{b}}$ \\
\hline
\end{tabular}

Tabla 5. Características de apariencia de los chorizos. Medias con el mismo superíndice en el mismo renglón no son significativamente diferentes $(p<0.05)$

\begin{tabular}{|c|c|c|c|c|}
\hline Atributos & $\begin{array}{l}\text { Carnicerías } \\
(n=10)\end{array}$ & $\begin{array}{c}\text { Mercados } \\
\text { rurales }(n=10)\end{array}$ & $\begin{array}{l}\text { Centros comerciales } \\
(n=10)\end{array}$ & $\begin{array}{l}\text { Centrales de } \\
\text { abasto }(n=10)\end{array}$ \\
\hline \multicolumn{5}{|l|}{ Morfología } \\
\hline Diámetro & $2.99 \pm 0.30^{\mathrm{a}}$ & $2.48 \pm 0.23^{b}$ & $3.17 \pm 0.68^{\mathrm{a}}$ & $2.87 \pm 0.35^{a}$ \\
\hline Tamaño de grano & $5.60 \pm 151$ & $6.05 \pm 2.23$ & $6.30 \pm 2.02$ & $5.30 \pm 1.25$ \\
\hline \multicolumn{5}{|l|}{ Color Interno } \\
\hline$L^{*}$ & $43.42 \pm 3.83^{\mathrm{ab}}$ & $40.03 \pm 5.22^{b}$ & $38.81 \pm 5.33^{b}$ & $47.17 \pm 5.20^{a}$ \\
\hline$a^{*}$ & $19.89 \pm 3.33^{\mathrm{ab}}$ & $17.85 \pm 2.95^{\mathrm{b}}$ & $21.37 \pm 3.85^{\mathrm{a}}$ & $22.93 \pm 3.40^{a}$ \\
\hline$b^{*}$ & $17.79 \pm 4.58$ & $15.81 \pm 3.67$ & $15.19 \pm 4.97$ & $19.05 \pm 4.43$ \\
\hline \multicolumn{5}{|l|}{ Color externo } \\
\hline$L^{*}$ & $36.71 \pm 2.61^{\mathrm{b}}$ & $36.79 \pm 5.24^{b}$ & $36.66 \pm 3.15^{b}$ & $43.14 \pm 5.30^{a}$ \\
\hline$a^{*}$ & $15.64 \pm 5.69^{\mathrm{ab}}$ & $11.27 \pm 3.04^{b}$ & $1544 \pm 6.01^{\mathrm{ab}}$ & $19.83 \pm 4.27^{a}$ \\
\hline$b^{*}$ & $10.67 \pm 6.82$ & $7.30 \pm 2.32$ & $8.68 \pm 5.33$ & $12.98 \pm 5.06$ \\
\hline \multicolumn{5}{|c|}{ Análisis del perfil de textura } \\
\hline Dureza $(\mathrm{N})$ & $3.48 \pm 1.72^{b}$ & $8.69 \pm 9.87^{b}$ & $16.62 \pm 8.60^{\mathrm{a}}$ & $4.32 \pm 3.36^{b}$ \\
\hline Adhesividad (N) & $-0.42 . \pm .0 .23$ & $-0.33 \pm 0.18$ & $-0.37 \pm 0.30$ & $-0.68 \pm 0.64$ \\
\hline Cohesividad & $0.54 \pm 0.17$ & $0.55 \pm 0.18$ & $0.60 \pm 0.15$ & $0.54 \pm 0.20$ \\
\hline Elasticidad & $0.34 \pm 0.09$ & $0.36 \pm 0.22$ & $0.42 \pm 0.21$ & $0.41 \pm 0.18$ \\
\hline Masticabilidad (N) & $0.60 \pm 0.36^{b}$ & $1.07 \pm 1.00^{b}$ & $3.61 \pm 2.87^{a}$ & $0.98 \pm 0.94^{\mathrm{b}}$ \\
\hline
\end{tabular}

Color

En cuanto al color interno, el de la masa, teniendo en cuenta los valores medios encontrados en los distintos tipos de chorizo (Tabla 5), el parámetro de luminosidad ( $\left.L^{*}\right)$ estuvo entre 38 y 47 , el componente rojo $a^{*}$ entre 18 y 23 y el componente amarillo $b^{\star}$ entre 15 y 19. Por su parte, en el color externo los valores obtenidos de $L^{*}$ fueron de 36 a 43, $a^{*}$ entre 11 y 20 y b* entre 7 y 13 . En general, el color interno presentó más luminosidad que en el color externo. Este hecho es el esperado ya que la luminosidad depende de la humedad y el interior de los chorizos contiene mayor humedad que el exterior. También en el color interno los valores de $a^{*} \mathrm{y}^{*}$ son mayores que en el externo, lo que puede estar asociado a una oxidación de los pigmentos en superficie, que están en contacto con el oxígeno atmosférico. 
En diversos estudios sobre chorizo español (Ansorena, et al., 1997; Gimeno, et al., 2000; Gómez, et al., 2001) y mexicano (Quintero-Salazar, et al., 2011) en los que se ha estudiado el color bien sea del chorizo recién embutido o bien en el transcurso de su elaboración, se han observado valores de los parámetros de color interno, para L* entre 30 y 50, a* desde 14 hasta 26 y b* desde 9 a 19. Los valores medios de los parámetros de color interno encontrados en los chorizos mexicanos estarían dentro de estos rangos. Comparando el color entre tipos de chorizo, se observaron diferencias significativas en los parámetros estudiados $(P<0.05)$. Estas diferencias pueden ser debidas a diversos factores. Por una parte están aquellos relativos a la composición mayoritaria del embutido como la cantidad de humedad o grasa, que tienen una influencia directa y marcada sobre la luminosidad. También se pude atribuir a la naturaleza y cantidad de chile (pimiento) deshidratado, o en su caso pimentón (Gómez, et al., 2001; Revilla y Vivar, 2005), usado en la formulación.

En el chorizo mexicano se emplean frutos de Capsicum spp., de distintas variedades, como ingrediente, en cantidades aproximadas de 30 a $50 \mathrm{~g}$ por $\mathrm{kg}$, principalmente de chile guajillo, según figura en diversos manuales y formulaciones utilizadas por pequeños elaboradores y en centros docentes, algunos de ellos publicados en libros de literatura nacional (Guerrero y Arteaga, 1990; Guerrero, et al., 2002). Otro factor con efecto sobre el color es la adición o no de nitratos y nitritos, que ocasiona la formación de nitrosomioglobina (Santamaría, et al., 1992; Frey, 1995; Perez-Dubé y Andujar-Robles, 2000) y que son empleados principalmente en los chorizos de centro comercial y centrales de abasto, mientras que estos no son aditivos de uso común en chorizos que se elaboran y comercializan en mercados rurales o carnicerías de ciudades.

La característica más importante de un producto para que los consumidores lo seleccionen en base a la calidad son los atributos de apariencia: contenido de grasa y color. La grasa se percibe como negativa debido a los aspectos (mencionados anteriormente) asociados con la salud, pero se percibe de manera positiva por su asociación al sabor y textura del producto (Resurreccion, 2003). La aparición de colores extraños no asociados a las características del producto resultaría en su rechazo.

\section{Características de textura}

En la Tabla 5 se muestran los resultados obtenidos en el análisis de perfil de textura en los distintos tipos de chorizos analizados. En la textura del chorizo debe influir decisivamente la materia prima cárnica (la cantidad de carne, grasa, tejido conjuntivo), la eventual presencia de almidones o proteínas no cárnicas, el diámetro del embutido y el grado de secado. El papel de la textura en la calidad de los chorizos no es del todo claro y depende de la variedad de chorizo de que se trate (Gimeno, et al., 2000). Las diferencias en las muestras analizadas para cada tipo de chorizo se podrían atribuir a variaciones en los factores anteriormente mencionados, siendo probable que la humedad sea uno de los más implicados (Revilla y Vivar, 2005; González-Fernández, et al., 2006).

La dureza y consecuentemente la masticabilidad fueron los parámetros sobre los que se observaron diferencias significativas entre tipos de chorizo. Los chorizos de centros comerciales fueron los más duros $(P<0.05)$. Este tipo de chorizos presentó el doble de dureza respecto a los chorizos de mercados rurales y fueron 4 veces más duros que los chorizos de centrales de abasto y carnicerías, respectivamente. Esta diferencia está relacionada con el menor contenido en humedad de los chorizos de centros comerciales. La adhesividad, cohesividad y elasticidad no presentaron diferencias entre tratamientos. Por último, se observó una diferencia altamente significativa $(P<0.001)$ entre tipos de chorizo para el parámetro masticabilidad. Los chorizos de centro comercial presentaron los valores más elevados $(3.61 \mathrm{~N})$ en comparación con los demás (0.88 $\mathrm{N}$ en promedio). La masticabilidad en el chorizo mexicano es inferior a la de un embutido crudocurado, lo que puede ser debido a un menor tiempo de secado ( $y$ una menor dureza) en el primer tipo de embutido.

De acuerdo a lo visto en los resultados, con vistas a una mejor estructuración del mercado, a la protección de productos típicos artesanales y del consumidor (mayor información, prevención de fraudes, etc.), se podrían establecer varias categorías diferenciadas de chorizo como por ejemplo: el chorizo artesanal (en base al tipo medio del chorizo elaborado en carnicerías y medio rural), el chorizo seco (el de centros comerciales) y chorizo económico (para los de centrales de abasto). La regulación de las condiciones técnicas de comercialización del chorizo debería mejorarse. En este sentido se podrían establecer las siguientes categorías: chorizo fresco, chorizo fermentado, chorizo seco o madurado; siendo el primero aquél que se comercialice siempre en frío, el segundo aquél con un pH igual o inferior a 5,0 y el tercero el que tenga una $a_{w}$ igual o menor a 0.90, tanto en México como en otros países Latinoamericanos donde haya productos similares. 


\section{CONCLUSIONES}

El chorizo mexicano comercializado en el Estado de Hidalgo podría considerarse como un embutido que presenta importantes variaciones en su contenido de humedad, aunque la mayor parte pertenece al grupo de embutidos semisecos (humedades entre 40 y 50\%). También hay variaciones importantes en los valores de $\mathrm{pH}$ y $\mathrm{a}_{\mathrm{w}}$. No obstante, la mayoría de los chorizos se pueden considerar como embutidos fermentados ya que presentaron valores de $\mathrm{pH}$ próximos a 5.0 y con un grado de secado significativo por tener $\mathrm{a}_{\mathrm{w}}$ entre 0.90 y 0.95 . Estos valores que confieren a este tipo de embutidos estabilidad microbiológica a temperatura ambiente. En cualquier caso, de acuerdo a su procedencia (tipo de elaboración) los chorizos presentan diferencias claras en sus propiedades de composición (componentes mayoritarios, perfil de ácidos grasos, color) que los hacen característicos.

\section{REFERENCIAS}

Adams, M.R., Fermented fresh foods. En: Adams, M.R. (Coord.)., Progress in industrial microbiology. Elsevier, Amsterdam, Holanda, 159-198 (1986).

Aguirrezábal, M., Mateo, J., Domínguez, C., Zumalacárregui, J.M., Spanish paprika and garlic as source of compounds of technological interest for the production of dry fermented sausages. Sciences des Aliments, 18, 409-414 (1998).

Aldai, N., Nájera, A.I., Dugan, M.E.R., Celaya, R., Osoro, K., Characterisation of intramuscular, intermuscular and subcutaneous adipose tissues in yearling bulls of different genetic groups. Meat Science, 76 (4) 682-691 (2007).

Ansorena, D., De Peña, M.P., Astiasarán, I., Bello, J., Colour evaluation of chorizo de Pamplona, a Spanish dry fermented sausage: comparison between the CIE $L^{*} a^{*} b^{*}$ and the Hunter Lab systems with illuminants D 65 and C. Meat Science, 46 (4) 313-318 (1997).

AOAC, Official Methods of Analysis of AOAC International, Official method 950.46. Moisture in meat; Official method 991.36. Fat (crude) in meat and meat products; Official method 992.15. Crude protein in meat and meat products. Block digestion method; Official method 920.153. Ash in meat. En: Cunniff. P. (Coord.). Meat and Meat Products, vol. II. $16^{\text {th }}$ ed., Official methods of analysis of the AOAC INTERNATIONAL, Gaithersburg, Maryland, EEUU, 1-15 (Chapter 39) (1999).

Austria, V., y otros 5 autores., Composition and physicochemical characteristics of "chorizo" from the Hidalguense Huasteca region in México. In $52^{\text {nd }}$ International Congress of Meat Science and Technology. Dublin, Irlanda (2006).

Bourne, M.C., Texture profile analysis. Food Technology, 32, (7) 62-66 (1978).

Bruna, J.M., y otros 5 autores., Changes in selected biochemical and sensory parameters as affected by the superficial inoculation of Penicillium camemberti on dry fermented sausages. International Journal of Food Microbiology, 85 (1-2) 111-125 (2003).

Carrapiso, A.I., Timón, M.L., Petrón, M.J., Tejeda, J.F., García, C., In situ transesterification of fatty acids from Iberian pig subcutaneous adipose tissue. Meat Science, 56 (2) 159-164 (2000).

CFIA, Canadian Food Inspection Agency. HACCP Generic model: fermented smoked sausage. En: http://www.inspection.gc.ca/english/fssa/polstrat/haccp/smsaufum/smsaufumie.shtml Acceso: Junio 7 (2007).

Chang, S.J., y Chow, C.K.J., Consumption of fatty acids. En: Foods and their Health Application (3er edición). C.K. Chow (editor). Boca Raton, CRC Press, 545-559 (2008).

CMC, Consejo Mexicano de la Carne. Compendio Estadístico 2011 de la Industria Cárnica Mexicana (2011).

Demeyer, D., y otros 19 autores., Control of bioflavour and safety in fermented sausages: first results of a European project. Food Research International, 33, 171-180 (2000).

Escartin, E.F., Castillo, A., Hinojosa-Puga, A., Saldaña-Lozano, J., Prevalence of Salmonella in chorizo and its survival under different storage temperatures. Food Microbiology, 16, 479-486 (1999).

Feiner, G., Meat products handbook. Woodhead Publishing limited. Cambridge, Reino Unido (2006). 
Flores, J., Bermell, S., Curado de embutidos. Consecuencias de la acidificación y factores que afectan. Fleischwirtsch, Español, 2, 22-26 (1995).

Frey, W., Fabricación fiable de embutidos. Editorial Acribia. Zaragoza, España (1995).

Gimeno, O., Anzorena, D., Astiasarán, I., Bello, J., Characterization of chorizo de Pamplona: instrumental measurements of colour and texture. Food Chemistry, 69 (2) 195-200 (2000).

Gómez, R., Picazo, M.I., Alvarruiz, A., Pérez, J.I., Valera, D., Pardo, J.E., Influencia del tipo de pimentón en la pérdida de color del chorizo fresco. Alimentaria, 28 (323) 67-73 (2001).

González-Fernández, C., Santos, E.M., Rovira, J., Jaime, I., The effect of sugar concentration and starter culture on instrumental and sensory textural properties of chorizo-Spanish dry-cured sausage. Meat Science, 74, 467-475 (2006).

González-Tenorio, R. y otros 4 autores. Características microbiológicas de cuatro tipos de chorizo comercializados en el Estado de Hidalgo. Nacameh, 6 (2), 25-32 (2012a).

González-Tenorio, R., Fernández-Diez, A., Caro, I., Mateo, J., Comparative Assessment of the Mineral Content of a Latin American Raw Sausage Made by Traditional or Non-Traditional Processes. En: Atomic Absorption Spectroscopy. Muhammad Akhyar Farrukh (editor). InTech. http://www.intechopen.com/books/atomic-absorption-spectroscopy/comparative-assessment-of-the-mineralcontent-of-a-latin-american-raw-sausage-made-by-traditional-or Acceso: Diciembre 12 (2012b).

Guerrero, I., Arteaga, M.R., Tecnología de carnes: elaboración y preservación de productos cárnicos. Ed. Trillas. UAM, México (1990).

Guerrero, I., Pérez, M.L., Ponce, E., Curso práctico de tecnología de carnes y pescado. Universidad Autónoma Metropolitana. Unidad Iztapalapa. México (2002).

Guerrero, L., y otros 12 autores., Consumer-driven definition of traditional food products and innovation in traditional foods. A qualitative cross-cultural study. Appetite, 52, 345-354 (2009).

Hill, F., The solubility of intramuscular collagen in meat animals of various ages. Journal of Food Science. 31 161-166 (1976).

Incze, K., Raw fermented and dried meat products. Meat Science. 49 (1) S168-S177 (1998).

Kuri, V., Madden, R.H., Collins, M.A., Hygienic quality of raw pork and chorizo (raw pork sausage) on retail sale in Mexico city. Journal of Food Protection, 59, No. 2, 141-145 (1995).

Mateo, J., Domínguez, M.C., Aguirrezábal, M.M., Zumalacárregui, J.M., Taste compounds in chorizo and their changes during ripening. Meat Science, 44, 245-254 (1996).

Mateo, J., y otros 4 autores., Meat processing in Ibero-American countries: a historical view. En: Noronha $V$, T., Nijkamp, P., and Rastoin, J.L. (Coord.) Traditional food production and rural sustainable. A European challenge. Ashgate publishing limited. Farnham, Surrey, Reino Unido, 121-134 (2009).

Pérez, L., y otros 4 autores., Evaluación química y sensorial del chorizo tipo Pamplona, elaborado a partir de carne de Cerdo Pelón Mexicano y de Cerdo Mejorado. Veterinaria México, 30 (1) 33-40 (1999).

Pérez-Dubé, D., y Andújar-Robles, G. Cambios de coloración de los productos cárnicos. Revista Cubana de Alimentación y Nutrición, 14 (2) 114-123 (2000).

Prieto, B., y Carballo, J. El control analítico de la calidad en los productos cárnicos crudos curados. Ciencia y Tecnología Alimentaria, 5 (1) 112-120 (1997).

Quintero-Salazar, B., y otros 4 autores., Tipificación parcial de embutidos artesanales de la Ciudad de Toluca: Chorizo verde. Nacameh, 5 (1) 10-26 (2011).

Raes, K., y otros 5 autores., Effect of linseed feeding at similar linoleic acid levels on the fatty acid composition of double-muscled Belgian Blue young bulls. Meat Science, 66 (2) 307-315 (2004). 
Ramos, D., y otros 7 autores., Composición y características fisicoquímicas de la salchicha de cerdo tradicional elaborada en el Departamento de Tumbes (Perú) Anales Científicos de la Universidad Nacional Agraria La Molina (2011).

Resurreccion, A.V.A., Sensory aspects of consumer choices for meat and meat products. Meat Science, 66, $11-20$ (2003).

Reuter, H., La tecnología de embutidos en Alemania. Fleishwirtschaft Español, 2, $46-49$ (1981).

Revilla, I., y Vivar, A.M. The effect of different paprika types on the ripening process and quality of dry sausages. International Journal of Food Science and Technology, 40 (4) 411-417 (2005).

Reyes-García, M., y otros 4 autores., Tablas peruanas de composición de alimentos. Disponible en: http://www.ins.gob.pe/insvirtual/images/otrpubs/pdf/Tabla\%20de\%20Alimentos.pdf (2009). Acceso 14 de Mayo (2012).

Santamaría, I., Lizarraga, T., Astiasarán, I., Bello, J. Contribución al problema del desarrollo del color en el chorizo de Pamplona: comportamiento de nitritos, nitratos y pigmentos cárnicos. Alimentaria, 29 (229) 23-26 (1992).

Stiebing, A., y Rödel, W., Influence of relative humidity on the ripening of dry sausage. Fleischwirtschaft, 68 (10) 1287-1291 (1988).

Szcesniak, S.A., Classification of Textural Characteristics. Journal of Food Science, 28(4) 385-389 (1963).

USDA, U.S. Department of Agriculture, Agricultural Research Service. (2010). USDA National Nutrient Database for Standard Reference, Release 23. Nutrient Data Laboratory Home Page, http://www.ars.usda.gov/ba/bhnrc/ndl. (Acceso: Octubre 19 (2010).

Vösgen, W., Embutidos secos, métodos probados y nuevas técnicas de producción. Fleischwirtsch Español 1, 17-21 (1995).

Walker, y otros 4 autores, Public health implications of meat production and consumption. Public Health Nutrition, 8, 348-356 (2005).

Wood, J.D., y otros 7 autores., Effects of fatty acids on meat quality: a review. Meat Science, 66 (1) 21-32 (2003).

Wood, J.D., y otros 7 autores., Fat deposition, fatty acid composition and meat quality: a review. Meat Science, 78 (4) 343-358 (2008). 
